\title{
Growth of Bifidobacteria in Adults' Intestines on Oral Administration of Sugar Source, Pantethine and Riboflavin
}

\author{
Kouhei Yazawa, ${ }^{1 *}$ Akiko Nakajima ${ }^{1}$ and Zenzo Tamura ${ }^{2}$ \\ Department of Analytical Chemistry, Faculty of Pharmaceutical Sciences, University of Tokyo, \\ Hongo, Bunkyo-ku, Tokyo 113,1 and Keio University Hospital Pharmacy, \\ Shinanomachi, Shinjuku-ku, Tokyo $160^{2}$
}

(Received 24 December, 1983)

\begin{abstract}
Among 45 Bifidobacterium strains of adult origin, all required a sugar source (ex. lactose) and pantethine (or pantothenic acid), 35 strains required riboflavin, and 12 required thiamine as essential nutrients. Fifteen strains were able to grow on pantothenic acid as well as pantethine. Fewer than 9 strains required other nutrients. The sugar source (10 to $15 \mathrm{~g} /$ day), pantethine $(50 \mathrm{mg} /$ day) and riboflavin $(5 \mathrm{mg} /$ day) were orally administered to 2 healthy adults whose proportion of bifidobacteria in the intestinal florae was usually very low (less than $1 \%$ of total bacterial number). The sugar source was lactulose, raffinose or inulin. Pantethine and riboflavin were packed together in capsules and enteric-coated. Administration of both sugar source and vitamin capsules caused marked growth of bifidobacteria (more than $40 \%$ of total bacterial number) in both adults. Administration of the sugar source alone caused moderate growth of bifidobacteria $(13 \%)$, while administration of the vitamin capsules alone was ineffective. These results indicate that the sugar source is essential for growth of bifidobacteria in adults, as well as in infants, and that administration of pantethine and riboflavin is important.
\end{abstract}

Key words: Nutritional requirement; pantethine; riboflavin; sugar source; Bifdobacterium; lactulose; raffinose, inulin

A great deal of investigation has been carried out on how to grow bifidobacteria in bottle-fed infants. One aspect is the search for bifidus-factors, that is, certain kinds of specific and vitamin-like growth factors for bifidobacteria. Glucosamine-derivatives $(3,4,15)$, peptides $(12)$, pantethine-derivatives $(14,16)$, nucleotides $(7)$ etc. have been found, but these are not very effective when administered to bottle-fed infants. Another aspect is the search for the sugar source for intestinal bifidobacteria. Bifidobacteria require large quantities of sugar source, from which the bacteria obtain energy and produce acetic and lactic acid. Hirata (6) and Yoshioka (17) reported a lack of sugar source in bottle-fed infants. Powdered milk has been improved for growing bifidobacteria by means of enrichment of lactose and supplementation of oligosaccharides such as lactulose $(10,11)$ which are negligibly absorbed by man.

On the other hand, little information is available on the growth of bifidobacteria in adults' intestines, although the importance of bifidobacteria in adults is not less than in infants Therefore, we started to investigate the nutritional requirements of bifidobacteria of adult origin. We administered the sugar source, pantethine and riboflavin as the required nutrients to 2 healthy adults whose bifidobacterial counts in intestinal flora had 


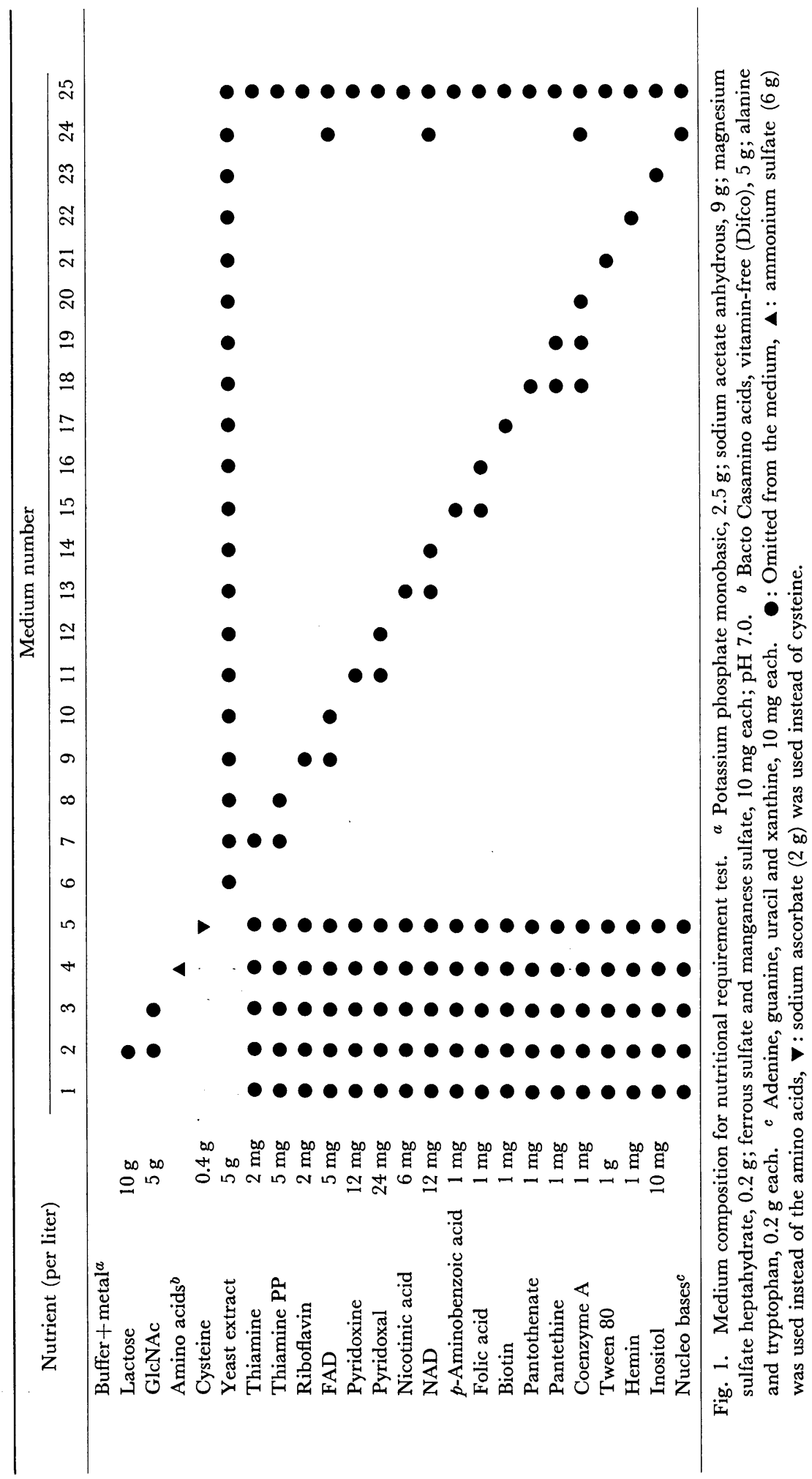


been very low. Simultaneously we tested which of sugar source and vitamin is the more important for growth of bifidobacteria.

\section{Materials and Methods}

1) Isolation of bifidobacteria: Feces from 22 adults (21 to 60 years) were diluted, smeared on BL-agar, BS-agar (9) and ALagar, and incubated using a steel wool method for 2 days. BL-agar and BS-agar were prepared using "BL-agar Eiken" (Eiken Kagaku, Tokyo). AL-agar was a selective medium for bifidobacteria of human origin, which grew well to form large swollen colonies. One liter of AL-agar contained $8 \mathrm{~g}$ of Bacto Casamino acids (Difco, Detroit, Michigan), $3.2 \mathrm{~g}$ of Polypepton (Daigo Eiyo, Osaka), $5 \mathrm{~g}$ of Bacto Yeast extract (Difco, Detroit, Michigan), $10 \mathrm{~g}$ of lactose, $1 \mathrm{~g}$ of $\mathrm{N}$-acetylglucosamine, $0.4 \mathrm{~g}$ of cysteine, $0.2 \mathrm{~g}$ of magnesium sulfate pentahydrate, $0.015 \mathrm{~g}$ of bromocresol green, $20 \mathrm{~g}$ of anhydrous sodium acetate, $5 \mathrm{ml}$ of $60 \%$ sodium lactate solution, and $15 \mathrm{~g}$ of agar. Bifidobacterial colonies on the agar plates were identified using Gram staining-microscopy, growth test in air, and the sugar fermentation test $(8)$. The colonies which showed very similar patterns to each other were collected into one strain. The forty-five strains isolated. consisted of $B$. bifidum (4), $B$. adolescentis (22) and B. longum (19).

2) Nutritional requirement test: The bifidobacterial strains were cultivated in Briggs liver broth (8) overnight at $37^{\circ} \mathrm{C}$ under an anaerobic atmosphere, washed twice with PBS containing $6 \mathrm{~mm}$ ascorbate by centrifugation, and suspended again in the PBS. A portion of the cell suspension was inoculated into such media, as certain nutrients had been omitted from the complete medium (medium number 1 to 25 as shown in Fig. 1), and incubated at $37^{\circ} \mathrm{C}$ for 4 days. When the strain showed no growth in the medium as assessed from the turbidity of the culture, the omitted nutrients were interpreted to be essential requirements of the strain.

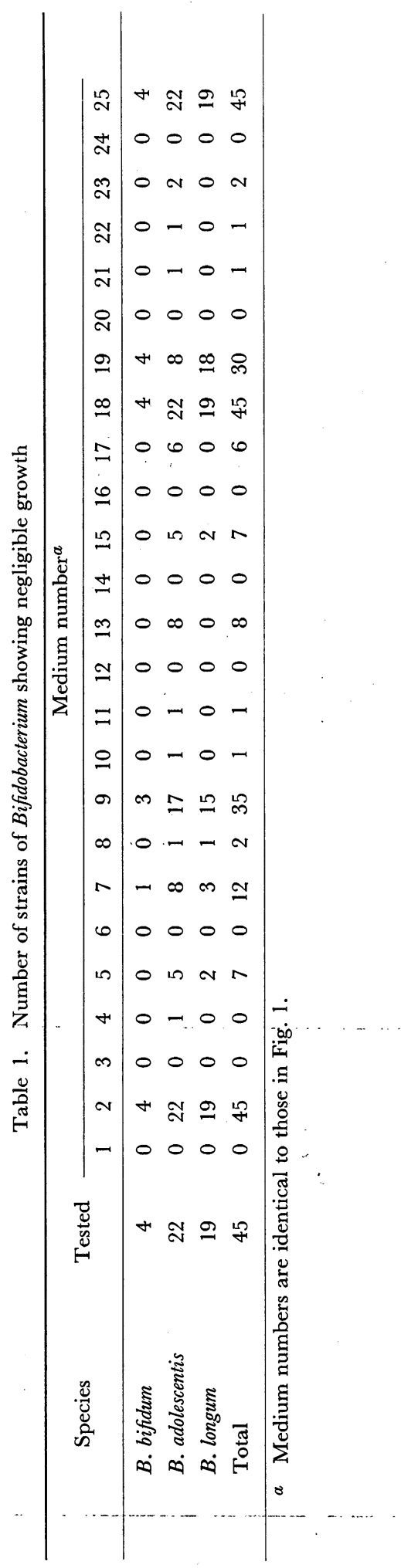


3) Oral administration experiment: Lactulose powder (Nikken Kagaku, Tokyo), raffinose (Wako Pure Chemicals, Osaka) or inulin (Yoneyama Kagaku, Osaka) was used as the sugar source for bifidobacteria. These three sugars are considered to be indigestible in human intestines $(1,13)$. Vitamin capsules were prepared through the courtesy of Daiichi Seiyaku Co., Tokyo. Each capsule contained $50 \mathrm{mg}$ of pantethine and $5 \mathrm{mg}$ of riboflavin, and was enteric-coated in order to prevent breakdown in the upper small intestines and to deliver the vitamins into the lower intestines. Two healthy male volunteers (32 and 60 years old) whose bifidobacteria counts in the intestinal flora were usually very low, were orally administered with 10 to $15 \mathrm{~g}$ of the sugar source per day in divided doses and/or one vitamin capsule per day after a meal. On the last day of each experimental period (about 10 days) feces were collected and their microflora were analyzed according to the method of Mitsuoka (9).

\section{Results and Discussion}

1) Nutritional requirement: As shown in Table 1, all of the bifidobacterial strains required the sugar source (ex. lactose, medium no. 2), but $\mathrm{N}$-acetylglucosamine was not essentially required (medium no. 3 ). Some of the strains $(27 \%)$ required thiamine but not thiamine pyrophosphate (media nos. 7 and 8). The great majority of the strains $(78 \%)$ required riboflavin but not FAD (media nos. 9 and 10). All of the strains required pantothenic acid or pantethine to make coenzyme A (media nos. 18, 19 and 20). In particular, pantethine was an essential requirement of almost all strains of $B$. bifidum and $B$. longum, while the majority of $B$. adolescentis strains grew on pantothenic acid. Very few or no strains required pyridoxine, nicotinic acid, folic acid, purines and pyrimidines.

Table 2. Change of fecal flora of subject Z. T. (60 years old)

\begin{tabular}{|c|c|c|c|c|c|}
\hline \multirow{4}{*}{ Organism } & \multicolumn{5}{|c|}{ Period } \\
\hline & -11.9 & -11.18 & -11.30 & -12.10 & -12.20 \\
\hline & \multicolumn{5}{|c|}{ Administration } \\
\hline & Non & $\begin{array}{l}\text { Lactulose } \\
\text { Vitamins }\end{array}$ & $\begin{array}{l}\text { Raffinose } \\
\text { Vitamins }\end{array}$ & Vitamins & Non \\
\hline Total count & $11.1^{a}$ & 11.2 & 11.1 & 11.2 & 11.0 \\
\hline Enterobacteriaceae & 8.5 & 6.9 & 8.0 & 8.2 & 7.8 \\
\hline Streptococcus & 9.2 & 9.4 & 9.4 & 8.8 & 9.1 \\
\hline Staphylococcus & 3.2 & 2.9 & 3.1 & 3.5 & 3.8 \\
\hline Yeast & 2.6 & nd & nd & 3.3 & nd \\
\hline Mold & $\mathrm{nd}^{b}$ & nd & nd & nd & nd \\
\hline Corynebacterium & nd & nd & nd & 5.2 & nd \\
\hline Bacillus & nd & 8.2 & nd & nd & nd \\
\hline Lactobacillus & 8.6 & 7.7 & 7.4 & 7.1 & 7.5 \\
\hline Bifidobacterium & 8.9 & 10.8 & 10.8 & 9.3 & 9.4 \\
\hline ( $\%$ of total count) & $(0.63)$ & $(40)$ & $(43)$ & $(0.99)$ & $(2.6)$ \\
\hline Eubacterium & 10.0 & 10.3 & 10.6 & 10.4 & 10.0 \\
\hline Bacteroidaceae & 11.0 & 10.9 & 10.5 & 11.0 & 10.8 \\
\hline Peptostreptococcus & 10.3 & nd & 9.9 & 10.6 & 10.6 \\
\hline Clostridium perfringens & 4.0 & nd & nd & 5.5 & nd \\
\hline Clostridium other & nd & nd & nd & nd & nd \\
\hline Veilonella & 6.9 & nd & 5.8 & 6.5 & 6.7 \\
\hline Other & nd & nd & nd & nd & nd \\
\hline
\end{tabular}

a $\log _{10}$ viable cell number/g feces.

$b$ Not detected. 
In the media containing yeast extract, casamino acids could be replaced by ammonium salt (medium no. 4), and cysteine by ascorbic acid (medium no. 5). This suggests that these amino acids were either not essential at all, or that they were contained sufficiently in the yeast extract. The requirement was estimated to be very small. Of the vitamins, biotin was required by only 6 strains (medium no. 17), differing from the results of Hassinen et al. (5) who investigated infant strains. At any rate, all or the vast majority of the bifidobacterial strains required the sugar source, pantethine and riboflavin.

The above results on vitamins are very similar to those of Gyllenberg and Gunnel (2) from infant strains of bifidobacteria. They reported that all of 20 tested strains required pantethine or pantothenic acid (in 12 strains pantethine was an essential requirement), 16 strains required riboflavin, and a few strains required biotin, thiamine, nico- tinic acid, purines and pyrimidines. They also reported that all amino acids except for cysteine were not essential but stimulatory, which on the whole agrees with our results.

2) Oral administration experiment: Two volunteers spent their everyday lives working, eating, drinking or sleeping. As shown in Table 2, administration of lactulose and the vitamin capsules (containing pantethine and riboflavin) increased markedly the proportion of bifidobacteria of the whole fecal bacterial number in the first volunteer from $0.65 \%$ to $40 \%$. Replacement of lactulose by raffinose did not change bifidobacterial levels $(43 \%)$. However, discontinuation of the sugar source decreased the bifidobacterial proportion to $0.99 \%$ in spite of continuation of vitamin administration. This shows the great importance of the sugar source for bifidobacterial growth in adults' intestines, as well as in infants' intestines. As for the second volunteer shown in Table 3, administration of lactulose and the vitamin

Table 3. Change of fecal flora of subject K. Y. (32 years old)

\begin{tabular}{|c|c|c|c|c|c|}
\hline \multirow{4}{*}{ Organism } & \multicolumn{5}{|c|}{ Period } \\
\hline & -11.9 & -11.18 & -11.30 & -12.10 & -12.20 \\
\hline & \multicolumn{5}{|c|}{ Administration } \\
\hline & Non & $\begin{array}{l}\text { Lactulose } \\
\text { Vitamins }\end{array}$ & $\begin{array}{l}\text { Inulin } \\
\text { Vitamins }\end{array}$ & Inulin & Non \\
\hline Total count & $11.3^{a}$ & 11.1 & 11.0 & 11.3 & 11.0 \\
\hline Enterobacteriaceae & 7.0 & 7.7 & 7.1 & 7.7 & 8.7 \\
\hline Streptococcus & 5.7 & 5.7 & 6.5 & 7.4 & 6.5 \\
\hline Staphylococcus & $\mathrm{nd}^{b}$ & nd & nd & nd & nd \\
\hline Yeast & nd & nd & nd & 2.9 & nd \\
\hline Mold & 2.2 & nd & nd & nd & nd \\
\hline Corynebacterium & 4.4 & nd & nd & nd & nd \\
\hline Bacillus & nd & 7.6 & nd & 7.7 & 7.4 \\
\hline Lactobacillus & nd & 3.6 & 3.6 & 2.5 & 4.0 \\
\hline Bifidobacterium & 8.6 & 10.9 & 10.6 & 10.4 & 8.6 \\
\hline ( $\%$ of total count) & $(0.25)$ & $(67)$ & $(41)$ & (13) & $(0.46)$ \\
\hline Eubacterium & 10.4 & 10.3 & 10.7 & 10.5 & 10.5 \\
\hline Bacteroidaceae & 11.0 & 10.1 & 9.4 & 11.0 & 10.7 \\
\hline Peptostreptococcus & 10.5 & 9.9 & 9.2 & 10.0 & 10.2 \\
\hline Clostridium perfringens & 4.5 & 2.9 & 4.7 & 3.5 & nd \\
\hline Clostridium other & nd & nd & nd & nd & nd \\
\hline Veilonella & 4.4 & 3.0 & nd & 3.2 & 3.9 \\
\hline Other & nd & nd & nd & nd & nd \\
\hline
\end{tabular}

a $\log _{10}$ viable cell number/g feces.

$b$ Not detected. 
capsules also increased the bifidobacterial count markedly from $0.25 \%$ to $67 \%$. Replacement of lactulose by inulin decreased it a little to $41 \%$, which is perhaps caused by the inability of bifidobacteria to utilize the larger molecular weight part to native inulin. Discontinuation of the vitamins but continuation of the sugar source (inulin) decreased the proportion of bifidobacteria to $13 \%$, the value is still 50 -fold more than that before administration. This indicates that administration of the sugar source alone is effective, and that administration of the sugar source, together with pantethine and riboflavine is very effective for intestinal bifidobacterial growth. Discontinuation of all administration decreased the count to $0.46 \%$. Changes of other bacterial numbers were not significant in these volunteers. Neither of them suffered from diarrhea or violent gas production, but felt rather better physically during the periods of administration of the sugar source plus the vitamins.

In the experiment, a dosage of sugar sources of 10 to $15 \mathrm{~g} /$ day was adopted, though the usual dosage of lactulose is 20 to $30 \mathrm{~g} /$ day. If the dosage was elevated in order to raise the bifidobacterial count to $100 \%$, diarrhea would possibly be experienced. Therefore, it is necessary to know the minimum effective dosage of respective sugar sources. From our results, the minimum dosage of certain ideal sugar sources, which are neither digested by man nor utilized by other intestinal bacteria except for bifidobacteria, seems to be about $5 \mathrm{~g}$ per day for adults. We have not tested smaller dosages of lactulose, raffinose or inulin along with the administration of vitamin capsules. Dosages of pantethine and riboflavin were not higher than those for usual treatment.

In conclusion, though the subjects (volunteers) numbered only two, administration of the sugar source together with pantethine and riboflavin was found to be very effective for growth of bifidobacteria in adults' intestines.

\section{Acknowledgements}

The authors wish to thank Mr. Naoyuki Inoue and Dr. Teruo Takayanagi, Daiichi Seiyaku Co., Tokyo, for the arrangements made to produce the enteric-coated capsules containing pantethine and riboflavin.

\section{References}

(1) Darlqvist, A. 1962. Specificity of human intestinal disaccharidases and implications of hereditary disaccharide intolerance. J. Clin. Invest. 41: 463-470.

(2) Gyllenberg, H., and C. Gunnel. 1958. The nutritional characteristics of the bifidobacteria (Lactobacillus bifidus) of infants. Acta Pathol. Microbiol. Scand. 44: 287-292.

(3) Gyöegy, P., R.F. Norris, and C.S. Rose. 1954. Bifidus factor. I. A variant of Lactobacillus bifidus requiring special growth factor. Arch. Biochem. Biophys. 48: 193-201.

(4) György, P., and C.S. Rose. 1955. Microbiological studies on growth factor for L. bifidus var. pennsylvanicus. Proc. Soc. Exp. Biol. N.Y. 90: 219223.

(5) Hassinen, J.B., G.T. Durbin, R.M. Tomarelli, and F.W. Bernhart. 1951. The minimal nutritional requirement of Lactobacillus bifidus. J. Bacteriol. 62: 771-777.

(8) Mitsuoka, T. 1974. Bacteriology of lactic acid bacteria. Rinsho Kensa 18: 1163-1172 (in Japanese).

(9) Mitsuoka, T. 1979. Normal intestinal microflora. Rinsho Kensa 23: 320-334 (in Japanese).

(10) Petuely, F. 1957. Bifidusflora bei Flaschenkindern durch bifidogene Substanzen (Bifidusfaktor). Z. Kinderheilk. 79: 174-179.

(11) Petuely, F. 1957. Der Bifidusfaktor. Dtsch. Med. Wschr. 82: 1959-1960.

(12) Raynaud, M. 1959. Le facteur Bifidus 2. Sem. Hôp. Paris 35: 240-255.

(13) Ruttloff, H., A. Täufel, W. Krause, H. Haenel, und K. Täufel. 1967. Die intestinal-enzymatische Spaltung von Galakto-Oligosacchariden in Darm von Tier und Mensch mit besonderer Berücksichtigung von Lactobacillus bifidus. Nahrung 11: $39-46$.

(14) Samejima, K., M. Yoshioka, and Z. Tamura. 1971. Bifidus factors in carrot. I. Chem. Pharm. Bull. (Tokyo) 19: 166-177.

(15) Tomarelli, R.M., J.B. Hassinen, E.R. Eckhardt, R.H. Clark, and F.W. Bernhart. 1954. The isolation of a crystalline growth factor for a strain of Lactobacillus bifidus. Arch. Biochem. Biophys. 48: 225-232. 
(16) Yoshioka, M., and Z. Tamura. 1971. Bifidus factors in carrot. II. Chem. Pharm. Bull. (Tokyo) 19: 178-185.
(17) Yoshioka, Y. 1971. Studies on Lactobacillus bifidus. Rep. Res. Lab., Snow Brand Milk Prod. Co., Ltd. (Tokyo) 72: 1-114. 